\title{
Differential immune responses to $\alpha$-gal epitopes on xenografts and allografts: implications for accommodation in xenotransplantation
}

\author{
Masahiro Tanemura, Dengping Yin, Anita S. Chong, and Uri Galili \\ Departments of Cardiovascular-Thoracic Surgery, General Surgery, and Immunology, Rush University, \\ Chicago, Illinois 60612, USA
}

Address correspondence to: Uri Galili, Xenotransplantation Research Laboratory, Department of CardiovascularThoracic Surgery, Rush University, 1653 West Congress Parkway, Chicago, Illinois 60612, USA.

Phone: (312) 942-7007; Fax: (312) 942-6862; E-mail: Uri_Galili@rush.edu.

Received for publication May 18, 1999, and accepted for publication December 14, 1999.

\begin{abstract}
Xenograft recipients produce large amounts of high-affinity anti-Gal IgG in response to Gal $\alpha 1$ 3Galß1-4GlcNAc-R ( $\alpha$-gal) epitopes on the graft. In contrast, ABO-mismatched allograft recipients undergo "accommodation," a state of very weak immune response to $\mathrm{ABO}$ antigens. These differences in anti-carbohydrate immune response were studied in $\alpha 1$,3galactosyltransferase knock-out mice. Pig kidney membranes administered to these mice elicited extensive production of anti-Gal IgG, whereas allogeneic kidney membranes expressing $\alpha$-gal epitopes elicited only a weak anti-Gal IgM response. Anti-Gal IgG response to xenograft membranes depended on helper T cell activation and was inhibited by anti-CD40L antibody. These $\mathrm{T}$ cells were activated by xenopeptides and not by $\alpha$-gal epitopes. Moreover, allogeneic cell membranes manipulated to express xenoproteins also induced anti-Gal IgG response. Xenoglycoproteins with $\alpha$-gal epitopes are processed by anti-Gal B cells. Xenopeptides presented by these cells activate a large repertoire of helper $\mathrm{T}$ cells required for the differentiation of anti-Gal B cells into cells secreting anti-Gal IgG. Alloglycoproteins with $\alpha$-gal epitopes have very few immunogenic peptides and fail to activate helper T cells. Similarly, ineffective helper T-cell activation prevents a strong immune response to blood group antigens in ABO-mismatched allograft recipients, thus enabling the development of accommodation.
\end{abstract}

J. Clin. Invest.105:301-310 (2000).

\section{Introduction}

The interaction between the natural anti-Gal antibody and the epitope Gal $\alpha 1-3 \mathrm{Gal} \beta 1-4 \mathrm{GlcNAc}-\mathrm{R}(\alpha-$ gal) on pig cells results in the rejection of pig xenografts in humans or monkeys (1-3). Hyperacute rejection of xenografts, mediated by anti-Gal IgM and complement, may be avoided by prevention of complement activation $(4,5)$. However, the detrimental effect of anti-Gal IgG molecules can be prevented only by removal of this antibody on affinity columns (6). This removal is temporary because anti-Gal reappears at its normal level after 5 to 6 days (6). Moreover, xenograft recipients produce large amounts of highaffinity anti-Gal IgG in response to $\alpha$-gal epitopes on the xenograft $(7,8)$. This elicited anti-Gal response induces a continuous inflammatory process that leads to chronic xenograft rejection (8). Development of methods aimed at preventing the elicited anti-Gal IgG production in xenograft recipients requires an understanding of the immune response to this carbohydrate epitope.

The information available regarding the immune response to carbohydrate epitopes on grafts is mostly limited to observations of the response to ABOincompatible allografts. Several studies have reported increased success of $\mathrm{ABO}$-incompatible allografts in splenectomized recipients in which anti-blood group antibodies were removed before transplantation (9-11). Rejection of more than $75 \%$ of such allografts was prevented despite the return of anti-blood group antibodies to normal levels. These observations led to the suggestion that the allograft cells undergo a process designated "accommodation," in which the cells may avoid rejection by expressing a variety of protective genes $(12,13)$. In contrast to the accommodation achieved toward ABO blood group epitopes on allografts, no accommodation toward $\alpha$-gal epitopes on xenografts has been reported, as yet. If human or monkey recipients are not completely immunosuppressed, the transplantation of pig xenografts results in an extensive increase in anti-Gal IgG activity and rejection of the xenograft $(7,8)$. This rejection raised the question whether there is a basic difference in the immune response to carbohydrate epitopes on allografts and xenografts. We studied this question in the experimental model of $\alpha 1,3$ galactosyltransferase $(\alpha 1,3 \mathrm{GT})$ knock-out (KO) mice (14). Our data imply that the extensive anti-Gal IgG production in response to $\alpha$-gal epitopes on xenografts is associated with the effective activation of helper $T$ cells by xenopeptides. 


\section{Methods}

Mice. KO mice for the $\alpha 1,3 \mathrm{GT}$ gene ( $\alpha 1,3 \mathrm{GT}$ KO mice) (14) were received from John B. Lowe and Aron Thall of the University of Michigan School of Medicine, Ann Arbor, Michigan, USA. The mice have the genetic background of $\mathrm{H}-2 \mathrm{~K}^{\mathrm{b}}, \mathrm{H}-2 \mathrm{~K}^{\mathrm{d}}, \mathrm{H}-2 \mathrm{D}^{\mathrm{b}}$, and $\mathrm{H}-2 \mathrm{D}^{\mathrm{d}}(129 \mathrm{SV} \times$ $\mathrm{DBA} / 2 \times \mathrm{C} 57 \mathrm{BL} / 6)$. The embryonic stem cells used were from $129 \mathrm{SV}$ mice $\left(\mathrm{H}-2 \mathrm{~K}^{\mathrm{b}}\right.$ and $\left.\mathrm{H}-2 \mathrm{D}^{\mathrm{b}}\right)$. Because these $\mathrm{KO}$ mice are not inbred, they have varying degrees of expression of $\mathrm{H}-2 \mathrm{~K}^{\mathrm{b}}$ and $\mathrm{H}-2 \mathrm{~K}^{\mathrm{d}}$ antigens on their cells. However, these mice completely lack $\mathrm{H}-2 \mathrm{~K}^{\mathrm{k}}$ and $\mathrm{H}-2 \mathrm{D}^{\mathrm{k}}$ antigens, which are present on immunizing allogeneic organs from $\mathrm{C} 3 \mathrm{H}$ mice. Kidneys or livers used for immunization of $\alpha 1,3 \mathrm{GT}$ KO mice were obtained from pigs, from wild type (WT) mice with similar $\mathrm{H}-2$ background (i.e., mice expressing $\alpha$-gal epitopes), and from the allogeneic $\mathrm{C} 3 \mathrm{H}$ mice, which also express $\alpha$-gal epitopes.

Immunization of mice. Kidney or liver fragments of mouse or pig origin were homogenized with a tissue homogenizer (30 seconds on ice). Membranes in the homogenates were washed 3 times by centrifugation for 30 minutes at 38,000 $\mathrm{g}$ and resuspended at a concentration of $250 \mathrm{mg} / \mathrm{mL}$ in PBS. Five-week-old mice were immunized intraperitoneally 3 times, in 2 -week intervals each, with $100 \mathrm{mg}$ membrane suspension.

Extraction of glycolipids for immunization. Glycolipids with $\alpha$-gal epitopes were extracted by Folch partition from $500 \mathrm{~mL}$ rabbit red blood cell (RBC) membranes, as a modification to a procedure we described previously (15). Rabbit RBC membranes where chosen as a source of glycolipids because almost all glycolipid molecules on these membranes with 5 or more carbohydrate units have terminal $\alpha$-gal epitopes $(15,16)$. These glycolipids, purified from the aqueous phase, were dried and dispersed in saline by sonication. Isolated glycolipids and pig kidney membrane homogenates were dried at various concentrations in the same ELISA plates. Relative expression of $\alpha$-gal epitopes in the 2 preparations was determined using ELISA with the anti-Gal mAb M86 (17). The glycolipid suspension was then diluted to contain the same $\alpha$-gal epitope concentration as in a pig kidney membrane suspension of $250 \mathrm{mg}$ membranes/mL. Mice were immunized 3 times intraperitoneally with glycolipids containing $\alpha$-gal epitopes in numbers equivalent to those in 100 $\mathrm{mg}$ of pig kidney membranes.

Measurement of anti-Gal production. Anti-Gal IgM and IgG production in response to immunization was determined in serum samples as described previously $(7,8,18)$ by ELISA, using synthetic $\alpha$-gal epitopes linked to BSA ( $\alpha$-gal-BSA, Dextra Laboratories, Reading, United Kingdom) as solid-phase antigen and HRP-coupled goat anti-mouse IgG or goat anti-mouse IgM as secondary antibodies (Axell Labs, Westbury, New York, USA).

Effect of anti-CD40L on anti-Gal production. Doses of 1 milligram of anti-mouse CD40L mAb produced in hamsters (19) (a gift from R.J. Noelle, Dartmouth University, Lebanon, New Hampshire, USA) were injected into mice tail veins. Anti-CD40L antibody was administered 1 day before immunization with $100 \mathrm{mg}$ pig kidney membranes on the day of immunization and 1 , 2 , and 3 weeks after this immunization. The mice also received a second immunization with these membranes 2 weeks after the first immunization. Anti-Gal production was determined 2 weeks after the second immunization. Control mice were immunized with pig kidney membranes and injected with hamster IgG in amounts comparable to the anti-CD40L antibody.

Effect of keybole limpet hemocyanin on anti-Gal response to allomembranes. The $\alpha 1,3 \mathrm{GT} \mathrm{KO}$ mice were immunized twice, intraperitoneally, in 2-week intervals, with $1 \mathrm{mg}$ keyhole limpet hemocyanin (KLH; Calbiochem-Novabiochem, San Diego California, USA), without adjuvant. After confirming production of anti-KLH by ELISA, the mice were further immunized twice with $100 \mathrm{mg} \mathrm{C} 3 \mathrm{H}$ kidney membranes that were coupled to KLH. Anti-Gal production was measured 2 weeks after the second immunization. Coupling of $\mathrm{KLH}$ to the membranes was performed by incubation of the membranes for 2 hours with $0.1 \% \mathrm{CrCl}_{3} \cdot 6 \mathrm{H}_{2} \mathrm{O}$ solution and with $10 \mathrm{mg} / \mathrm{mL}$ of KLH. Unbound KLH was removed by repeated washing of the membranes, and the coupling was confirmed by the binding of anti-KLH antibodies to the membranes that were used as solid-phase antigen in ELISA.

Flow cytometry analysis of anti-Gal B cells. B cells capable of producing anti-Gal (i.e., expressing $B$ cell receptors [BCR] that bind $\alpha$-gal epitopes, designated here as anti-Gal B cells) were identified by their ability to bind $\alpha$-gal-BSA. Mouse splenocytes $\left(10^{6}\right.$ cells $)$ were incubated for 2 hours at $4{ }^{\circ} \mathrm{C}$ with biotinylated $\alpha$ - galBSA $(1 \mu \mathrm{g} / \mathrm{mL})$ in PBS containing $1 \%$ BSA and subsequently with avidin-FITC and phycoerythrin-conjugated (PE-conjugated) anti-mouse Ig (PharMingen, San Diego, California, USA) for double-color analysis of B cells. The stained cells were analyzed in a FACScalibur flow cytometer (Becton Dickinson Immunocytometry Systems, San Jose, California, USA). Biotinylation of $\alpha$-gal BSA was performed with the biotin long-chain-NHS compound (Pierce Chemical Co., Rockford, Illinois, USA) according to the manufacturer's instructions. Similar staining was performed with biotinylated $N$-acetyllactosamineBSA that was obtained by treatment of $\alpha$-gal-BSA with recombinant $\alpha$-galactosidase (8).

Immunization with bybridomas. Manipulation of mouse cells to express xenoproteins was achieved by the formation of human/mouse hybridoma. Mouse/mouse and human/mouse polyclonal hybridomas were generated by fusion of $2 \times 10^{7}$ mouse SP2/0 myeloma fusion partner cells with an equal number of $\alpha 1,3 \mathrm{GT} \mathrm{KO}$ mouse splenocytes or human blood lymphocytes and subsequent culturing of the fused cells in HAT medium. The nonfused mouse or human lymphocytes, as well as SP2/ 0 cells, die within 1 to 2 weeks of culturing. The $\alpha 1,3 \mathrm{GT}$ KO mice were immunized intraperitoneally 3 times with $2 \times 10^{6}$, followed by $20 \times 10^{6}$ and 
$18 \times 10^{6}$ mouse/mouse $\left(10^{6} \alpha\right.$-gal epitopes/cell $)$ or human/mouse hybridoma cells $\left(0.25 \times 10^{6} \alpha\right.$-gal epitopes/cell), with 2 -week intervals between the immunizations. Anti-Gal IgG production was determined 2 weeks after the third immunization.

Transfection of cells with $\alpha 1,3 G T$ gene. Two types of cells that lack $\alpha$-gal epitopes, mouse BL6 melanoma and African green monkey COS cells, were transfected with mouse $\alpha 1,3 \mathrm{GT}$ cDNA (20) to induce stable expression of $\alpha$-gal epitopes, as we have described previously (21). The 2 transfected cell lines, designated here $B L 6_{\alpha G T}$ and $\operatorname{COS}{ }_{\alpha \mathrm{GT}}$ were found to express $2.0 \times$ $10^{6}$ and $10^{6} \alpha$-gal epitopes/cell, based on analysis by the ELISA inhibition assay (17).

In vitro stimulation of lymphocytes. To determine whether splenocytes from mice immunized with pig kidney membranes can be directly stimulated by $\alpha$ gal epitopes, an in vitro cell proliferation assay was performed. The pig kidney cell line, PK15, was also used as stimulatory cells. The same cells, however, devoid of $\alpha$-gal epitopes by treatment with $10 \mathrm{U} / \mathrm{mL}$ recombinant $\alpha$-galactosidase (8), were studied as stimulatory cells, as well. Other cells used as stimulatory cells were Old World monkey COS cells, $\mathrm{COS}_{\alpha \mathrm{GT}}$ cells expressing $\alpha$-gal epitopes, mouse BL6 melanoma cells lacking $\alpha$-gal epitopes, and BL $6_{\alpha \mathrm{GT}}$ cells expressing this epitope (21). All types of stimulatory cells were irradiated with 50 Gy and frozen until used. In addition, pig kidney membranes treated with $\alpha$-galactosidase or untreated membranes were assayed for their stimulatory effect.

Mouse splenocytes $\left(2 \times 10^{5}\right.$ cells/well $)$ in RPMI1640 medium supplemented with $10 \%$ FCS and 50 $\mu \mathrm{M}$ of 2 -mercaptoethanol were incubated in $0.2-\mathrm{mL}$ aliquots with $10^{4}$ stimulatory cells, pig kidney membranes $(10 \mu \mathrm{g} /$ well $)$, or with glycolipids expressing $\alpha$ gal epitopes at a concentration range equivalent to concentration of these epitopes on $10 \mu \mathrm{g} / \mathrm{mL}$ to 1 a

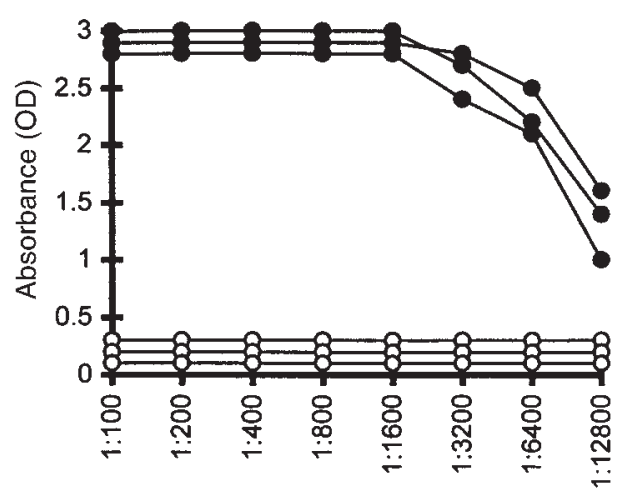

Serum dilution

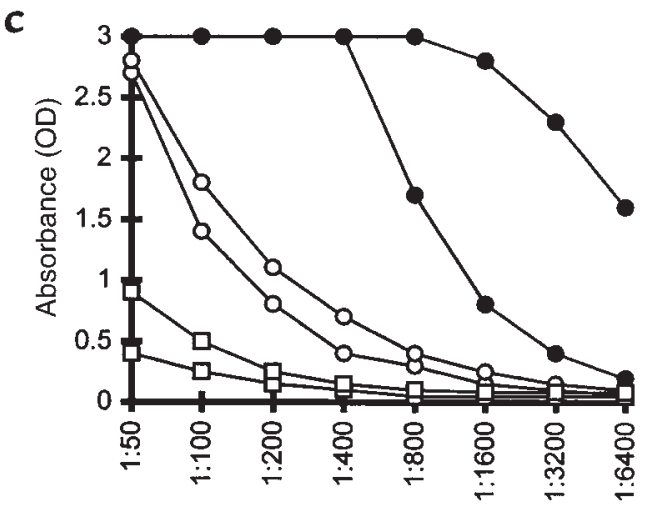

Serum dilution b

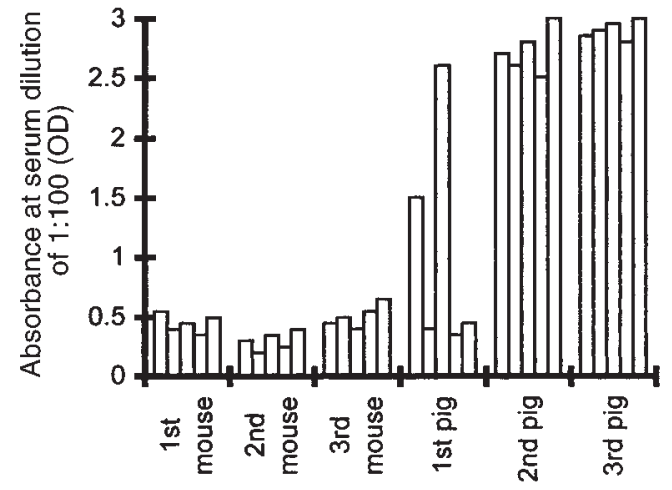

Origin of immunizing kidney membranes

d

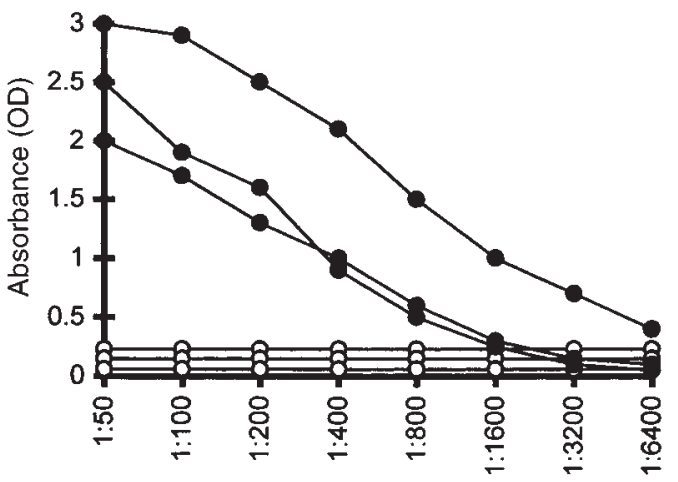

Serum dilution

Figure 1

(a) Anti-Gal IgG production in $\alpha 1,3 \mathrm{GT} \mathrm{KO}$ mice immunized 3 times with kidney membranes from pig (filled circles) or C3H mouse (open circles). Data represent anti-Gal activity in 3 characteristic mice of 8 in each group with similar results. (b) Anti-Gal IgG activity in 5 mice immunized first 3 times with WT mouse kidney membranes followed by 3 immunizations with pig kidney membranes. Data represent absorbance 2 weeks after each immunization at serum dilution of 1:100. (c) Anti-Gal IgM response in nonimmunized $\alpha 1,3 G T$ KO mice (open squares), mice immunized 3 times with pig kidney membranes (filled circles), or with WT mouse kidney membranes (open circles). Data from 2 representative mice from groups of 5 animals per group. (d) Anti-Gal IgM (filled circles) and IgG (open circles) response in mice immunized 3 times with glycolipids containing $\alpha$-gal epitopes in amounts equivalent to the amount of this epitope in 100 mg pig kidney membranes. Data are from 3 representative mice of 6 per group with similar results. 


\section{Figure 2}

Thin-layer chromatography separation of rabbit RBC glycolipids extracted in the organic (lane 1) and aqueous phase (lane 2) after Folch partition. Glycolipids were stained with orcinol. Note that the organic phase contains primarily ceramide trihexoside $(\mathrm{CTH})$ with the structure Gal 1 1-4Galß1-4Glc-Cer, whereas the aqueous phase contains mostly $\mathrm{CPH}$ with the structure Gal $\alpha 1$ 3Gal $\beta 1$ - 4GlcNAc $\beta 1-3 G a l \beta 1-4 G l c-$ Cer, and long-chain glycosphingolipids (LC-GSL), all of which have $\alpha$-gal epitopes $(15,16)$

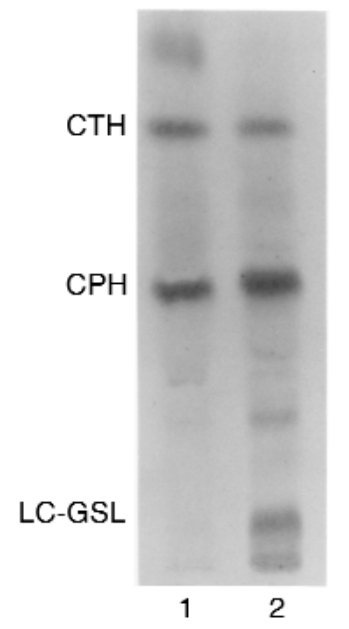

$\mathrm{mg} / \mathrm{mL}$ of pig kidney membranes. After 5 days of incubation at $37^{\circ} \mathrm{C}, 1.0 \mu \mathrm{Ci}$ of $\left[{ }^{3} \mathrm{H}\right]$ thymidine was added to each well. Cells were harvested after additional incubation for 24 hours and $\left[{ }^{3} \mathrm{H}\right]$ thymidine uptake into the cells was determined.

Antibody-dependent cell-mediated cytotoxicity assay. The antibody-dependent cell-mediated cytotoxicity (ADCC) assay was performed as described previously (1). Various target cells were plated (100 cells/well) in Terasaki plates. Subsequently, $\alpha 1,3 \mathrm{GT}$ KO mouse splenocytes were added to the wells at various effector/target cell ratios in $20-\mu \mathrm{L}$ aliquots of DMEM supplemented with $10 \%$ FCS and in presence or absence of $4 \mu \mathrm{g} / \mathrm{mL}$ of mouse anti-Gal. The plates were incubated for 24 hours at $37^{\circ} \mathrm{C}$, then washed for removal of killed cells, which detach from the wells. The plates were stained with

a

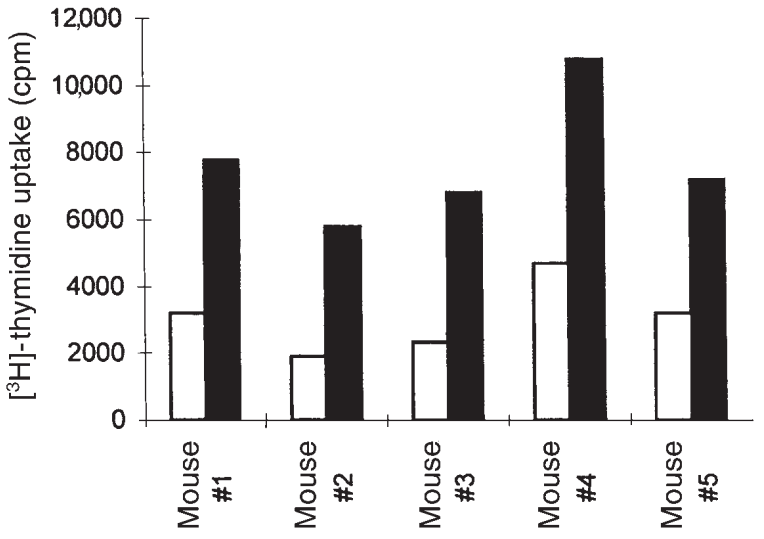

Wright stain and inspected microscopically to determine the proportion of killed cells. Mouse anti-Gal used in these assays was isolated by affinity chromatography from sera of $\alpha 1,3 \mathrm{GT}$ KO mice immunized with pig kidney membranes, as described previously (18).

\section{Results}

Anti-Gal IgG response to xenomembranes and allomembranes. $\alpha 1,3 \mathrm{GT}$ KO mice were immunized 3 times, intraperitoneally, with $100 \mathrm{mg}$ of allomembranes isolated from C3H kidney homogenates or with $100 \mathrm{mg}$ pig kidney membranes. The elicited production of anti-Gal in the immunized mice was assayed by ELISA using synthetic $\alpha$-gal epitopes coupled to BSA as solid-phase antigen (18). As shown in Figure 1a, immunization with pig kidney membranes resulted in extensive production of antiGal IgG. This antibody was detectable at serum dilutions well above 1:10,000. A similar response was observed when the mice were immunized with $1 \mathrm{mg}$ instead of $100 \mathrm{mg}$ pig membranes (not shown). This strong antiGal IgG response was also observed in mice that were splenectomized before immunization with $100 \mathrm{mg}$ of pig kidney membranes (not shown). In contrast, no antiGal IgG production was detected when mice were immunized 3 times with $100 \mathrm{mg}$ of $\mathrm{C} 3 \mathrm{H}$ mouse kidney membranes (Figure 1a). A similar differential anti-Gal IgG response was observed in mice immunized with liver membranes. Three immunizations with $100 \mathrm{mg}$ of pig liver membranes elicited a strong anti-Gal IgG response, whereas immunizations with mouse liver membranes did not elicit such an antibody response (not shown).

Immunization with WT mouse kidney membranes (i.e., mice producing $\alpha$-gal epitopes and expressing MHC molecules similar to the $\alpha 1,3 \mathrm{GT}$ KO mice) also did not produce anti-Gal IgG (Figure 1b). This lack of

b

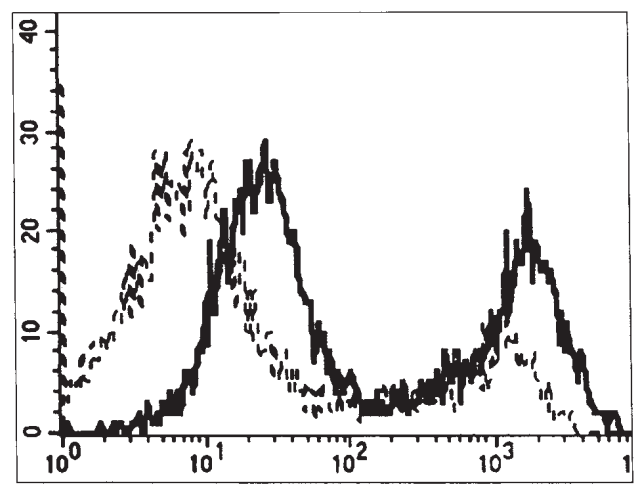

Source of lymphocytes for MLR

Figure 3

Allogeneic response of $\alpha 1,3 \mathrm{GT}$ KO mice to $\mathrm{C} 3 \mathrm{H}$ mice. (a) Stimulation of lymphocytes from $5 \alpha 1,3 \mathrm{GT}$ KO mice in a MLR with irradiated C3H mouse lymphocytes as stimulatory cells (closed columns) or in the absence of stimulatory cells (open columns). (b) Production of alloantibodies in $\alpha 1,3 \mathrm{GT}$ KO mice immunized with $\mathrm{C} 3 \mathrm{H}$ kidney membranes; FACS analysis of C3H spleen lymphocytes incubated with sera from nonimmunized $\alpha 1$, 3GT KO mice (broken line), or sera from immunized mice (solid line). The left peak in each histogram represents $T$ cells, whereas the right peak represents $B$ cells. Data are from 1 of 5 mice with similar results. 
anti-Gal IgG response was not due to tolerance induction toward $\alpha$-gal epitopes on the mouse kidney membranes. This is indicated by the finding that subsequent immunizations of these mice with pig kidney membranes resulted in an extensive anti-Gal IgG production, similar to that observed in naive $\alpha 1,3 \mathrm{GT}$ KO mice immunized with pig kidney membranes (Figure 1b).

Whereas $\alpha$-gal epitopes on mouse kidney membranes failed to induce anti-Gal IgG production (Figure 1a), the interaction of these epitopes with the BCR on the corresponding B cells was sufficient for the induction of a relatively weak anti-Gal IgM response. As shown in Figure 1c, activity of anti-Gal IgM is very low in nonimmunized mice. Three immunizations of the $\alpha 1,3 \mathrm{GT}$ KO mice with $100 \mathrm{mg}$ WT mouse kidney membranes, resulted in some increase in the activity of anti-Gal IgM antibodies. Similar immunization with pig kidney membranes resulted in a much higher activity of this antibody.

The strong anti-Gal IgG response in $\alpha 1,3 \mathrm{GT}$ KO mice immunized with pig kidney membranes is associated with an immune response to xenoglycoproteins expressing $\alpha$-gal epitopes. Glycolipids with this epitope are not capable of inducing this response. Such glycolipids were isolated from large amounts of rabbit RBC membranes by extraction and Folch partition. The aqueous hydrophilic phase in Folch partition of these membranes contained primarily ceramide pentahexoside $(\mathrm{CPH})$ and ceramides with longer carbohydrate chains that also have $\alpha$-gal epitopes (lane 2 in Figure 2; refs. 15, 16). These glycolipids were dispersed in saline as micelles, and their concentration was adjusted to contain the same amount of $\alpha$-gal epitopes as that in $250 \mathrm{mg} / \mathrm{mL}$ of pig kidney membranes. Mice were immunized 3 times with a suspension of glycolipid containing $\alpha$-gal epitopes in an amount equivalent to that of this epitope in $100 \mathrm{mg}$ pig kidney membranes. Analysis of the sera from these mice demonstrated a weak anti-Gal IgM production and the complete lack of anti-Gal IgG (Figure 1d).

Allogeneic response of $\alpha 1,3 \mathrm{GT} \mathrm{KO}$ mice to $\mathrm{C} 3 \mathrm{H}$ mice. The lack of anti-Gal IgG response in $\alpha 1,3 \mathrm{GT} \mathrm{KO}$ mice immunized with $\mathrm{C} 3 \mathrm{H}$ mouse kidney membranes was not a result of insufficient allogeneic response. As shown in Figure 3a, $\alpha 1,3$ GT KO mice displayed an effective mixed lymphocyte reaction (MLR) when incubated with irradiated $\mathrm{C} 3 \mathrm{H}$ splenocytes. In addition, immunization of $\alpha 1,3 \mathrm{GT}$ KO mice with kidney membranes from $\mathrm{C} 3 \mathrm{H}$ mice resulted in the production of alloantibodies that readily bound to $\mathrm{C} 3 \mathrm{H}$ lymphocytes. As shown by FACS analysis, antibodies in sera of the immunized mice readily bound to $T$ cells, whereas sera from nonimmunized mice lacked this antibody activity (left peaks in Figure 3b). The staining of B cells in these populations was almost maximal with the secondary antibody (right peak), because of binding to cell-surface Ig molecules. It should be stressed that these sera were depleted of anti-Gal by adsorption on pig kidney membranes before incubation with $\mathrm{C} 3 \mathrm{H}$ lymphocytes.
Anti-Gal B cells in immunized mice. The marked increase in anti-Gal IgG titers in mice immunized with pig kidney membranes was associated with an extensive expansion of B-cell clones with anti-Gal BCR (designated here as anti-Gal B cells), whereas no such expansion was found in mice immunized with mouse kidney membranes that express $\alpha$-gal epitopes or with glycolipids expressing this epitope. Identification of anti-Gal $\mathrm{B}$ cells was achieved by measuring the interaction between B cells and synthetic $\alpha$-gal epitopes coupled to biotinylated BSA ( $\alpha$-gal BSA) and subsequent binding of FITC-avidin. B cells were immunostained by PE-coupled anti-mouse Ig. As shown in Figure 4a, nonimmunized $\alpha 1,3 \mathrm{GT}$ KO mice had relatively few anti-Gal B cells. However, subsequent to immunization with pig kidney membranes, the proportion of these cells increased by severalfold (Figure 4b). The specificity of this interaction was indicated by the finding that destruction of the $\alpha$-gal epitopes with $\alpha$-galactosidase

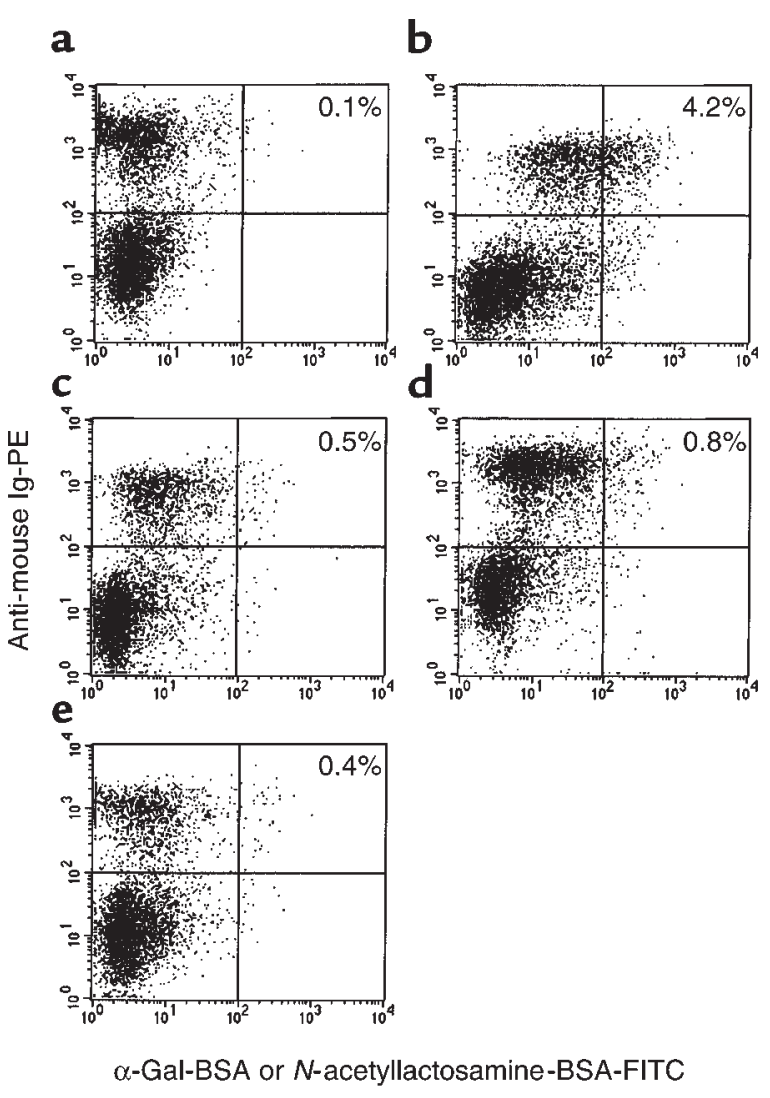

Figure 4

Two-color FACS analysis of B cells with anti-Gal BCR in $\alpha 1,3 \mathrm{GT}$ KO mice. $B$ cells were identified by the staining with $P E$-conjugated antimouse Ig, whereas cells with anti-Gal BCR were identified by the binding of biotinylated $\alpha$-gal BSA, followed by avidin-FITC. The stained lymphocytes were from: (a) nonimmunized mice; (b) mice immunized with pig kidney membranes; (c) cells from mice immunized as in $\mathbf{b}$ and tested for binding of biotinylated $N$-acetyllactosamine (Gal $\beta 1-4 \mathrm{Glc}-$ NAc-R)-BSA as specificity control; (d) mice immunized with WT mouse kidney membranes; (e) mice immunized with glycolipids from rabbit RBC. Proportion of double-positive stained cells is indicated. Data are from a representative mouse of 5 in each group. 


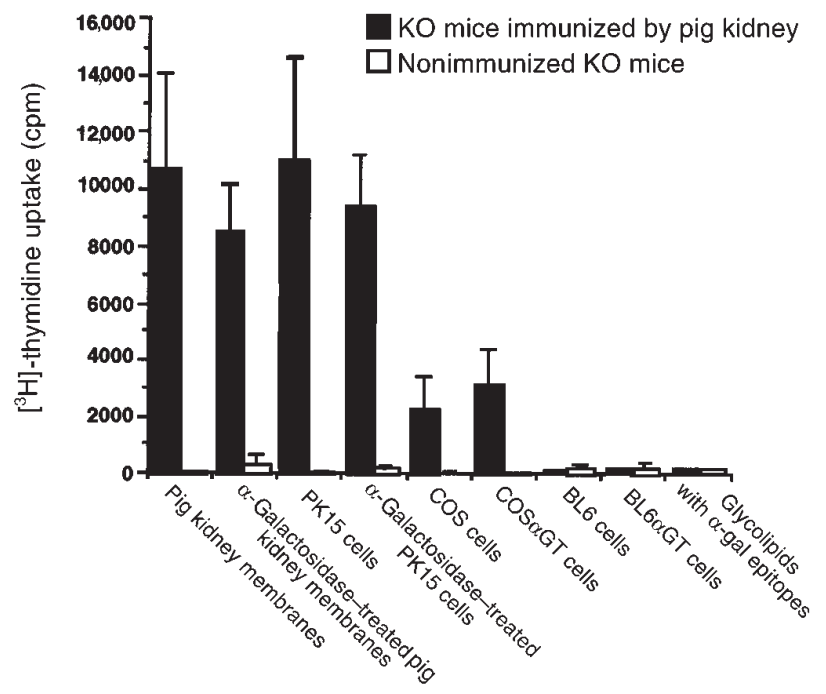

Figure 5

Effect of $\alpha$-gal epitope expression on in vitro proliferation of spleen lymphocytes from nonimmunized $\alpha 1,3 \mathrm{GT} \mathrm{KO}$ mice (open columns) or from $\alpha 1,3 G T$ KO mice immunized 3 times with pig kidney membranes (closed columns). Pig kidney membranes or pig PK15 cells were treated with $\alpha$-galactosidase for the removal of $\alpha$-gal epitopes. $\operatorname{COS} \alpha$ GT and BL $6_{\alpha \text { GT }}$ cells are transfected with mouse $\alpha 1,3 G T$ cDNA and express $\alpha$-gal epitopes. Glycolipids extracted from rabbit RBC were assayed at concentrations that corresponded to $\alpha$-gal epitope expression by $100 \mu \mathrm{g} / \mathrm{mL}$ of pig kidney membranes. Data are mean \pm $\mathrm{SE}$ of from 5 mice in each group.

resulted in the subsequent elimination of $\alpha$-gal BSA binding by $\mathrm{B}$ cells from the immunized mice (Figure $4 c)$. The $\alpha 1,3$ GT KO mice immunized with WT mouse kidney membranes expressing $\alpha$-gal epitopes displayed only a minor increase in the proportion of anti-Gal B cells (Figure 4d). Similarly, mice immunized with glycolipids that have $\alpha$-gal epitopes did not display a significant expansion of anti-Gal B cells (Figure 4e).

Lack of significant T-cell response to $\alpha$-gal epitopes on xenografts. The studies above demonstrate the need for immunization of $\alpha 1,3 \mathrm{GT}$ KO mice with xenomembranes to induce significant expansion of anti-Gal Bcell clones and isotype switch, so that anti-Gal IgG can be produced. Because isotype switch is a T celldependent process (22), it was of interest to determine whether helper $T$ cells can be specifically activated by $\alpha$-gal epitopes expressed on xenoglycoproteins. For this purpose we studied the proliferation of lymphocytes from $\alpha 1,3 \mathrm{GT}$ KO mice immunized with pig kidney membranes in the presence of xenograft cell membranes expressing or lacking $\alpha$-gal epitopes. As shown in Figure 5, such lymphocytes were induced to proliferate in the presence of these membranes. However, only a minor decrease in the proliferation rate was observed in the absence of $\alpha$-gal epitopes, i.e., in cultures containing $\alpha$-galactosidase-treated membranes. The observed proliferation was likely to result from specific immune response to xenopeptides in the kidney membrane fragments, because lympho- cytes from nonimmunized mice displayed almost no proliferative response when incubated with these membranes. Lymphocytes from immunized mice also were stimulated to proliferate by the pig kidney cell line PK15. However, no significant difference in the proliferative response was observed in cultures that contained irradiated $\alpha$-galactosidase-treated PK15 cells that lacked $\alpha$-gal epitopes.

Stimulation was also tested with irradiated monkey COS cells that normally lack $\alpha$-gal epitopes or with $\operatorname{COS}{ }_{\alpha G T}$ cells expressing $2 \times 10^{6} \alpha$-gal epitopes/cell as a result of stable transfection with mouse $\alpha 1,3 \mathrm{GT}$ cDNA. Both types of COS cells displayed a similar stimulatory effect on $\alpha 1,3 \mathrm{GT}$ KO mouse lymphocytes. Interestingly, the proliferation induced by these monkey cells was significantly lower than that induced by the pig kidney membranes or by PK15 cells. This further suggests that a significant proportion of the stimulatory effect is due to specific recognition of pig peptide antigens present in pig kidney membranes and in PK15 cells, but absent in monkey cells. Lymphocytes from immunized $\alpha 1,3 \mathrm{GT}$ KO mice failed to proliferate when incubated with mouse BL6 ${ }_{\alpha \mathrm{GT}}$ cells expressing $10^{6} \alpha$-gal epitopes/cell or with BL6 cells lacking this epitope (17). This failure to proliferate further supports the assumption that this epitope does not effectively stimulate $T$ cells in immunized animals. Accordingly, glycolipids with $\alpha$-gal epitopes also were found to lack a stimulatory effect. In all these assays, the lymphocytes were viable and competent because they effectively proliferated as a result of nonspecific stimulation by phytohemagglutinin (PHA) (not shown).

Anti-Gal response is dependent on helper T cells activated by xenopeptides. The lack of significant proliferative T-cell response that is specific to $\alpha$-gal epitopes raised the question whether anti-Gal IgG response in xenograft recipients is T-cell dependent. This question was studied by evaluating anti-Gal response in mice immunized with pig kidney membranes and treated with the hamster anti-CD40L mAb, which prevents T-B cell interaction (19). As shown in Figure 6a, repeated delivery of this antibody, in the course of 2 immunizations with pig kidney membranes, completely prevented anti-Gal IgG production. In contrast, mice treated with comparable amounts of hamster IgG, readily produced antiGal IgG when immunized with pig membranes. Interestingly, anti-Gal IgM production in the immunized mice was not affected by the anti-CD40L antibody.

The observations on differential anti-Gal IgG response to $\alpha$-gal epitopes on xenograft and allograft membranes (Figure 1), the lack of specific proliferative T-cell response to $\alpha$-gal epitopes (Figure 5), and the T-cell dependent anti-Gal IgG response to xenografts (Figure 6a) all suggested that this response is associated with the activation of helper T cells by xenopeptides, rather than by $\alpha$-gal epitopes. Therefore, we have determined whether manipulation of mouse cells expressing $\alpha$-gal epitopes to express xenoproteins would confer on such cells the immunogenic potential to induce anti-Gal IgG production. For 
a

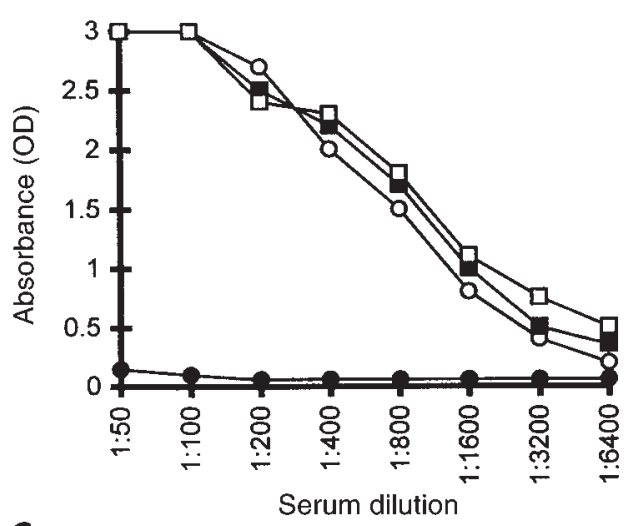

c

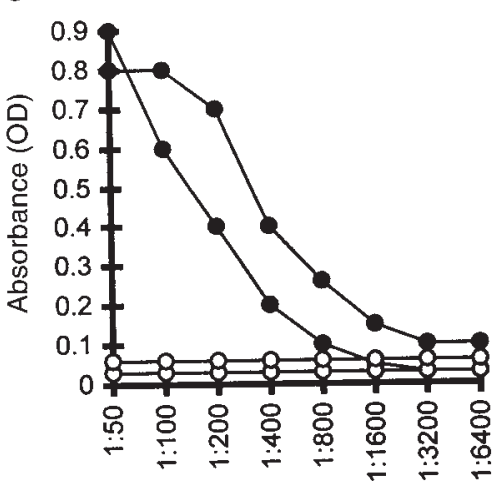

Serum dilution b

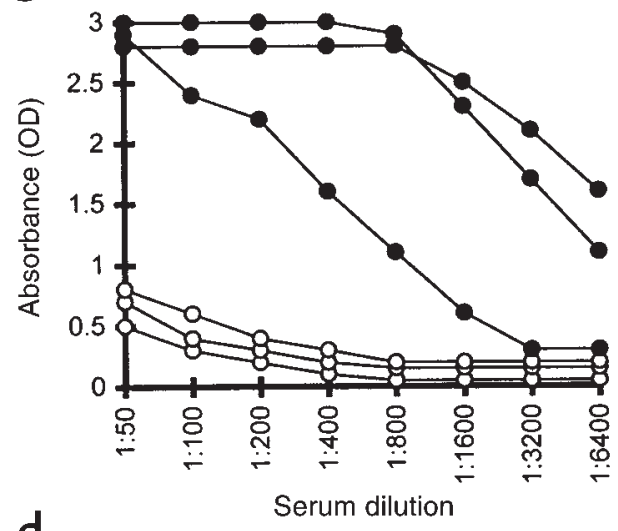

d

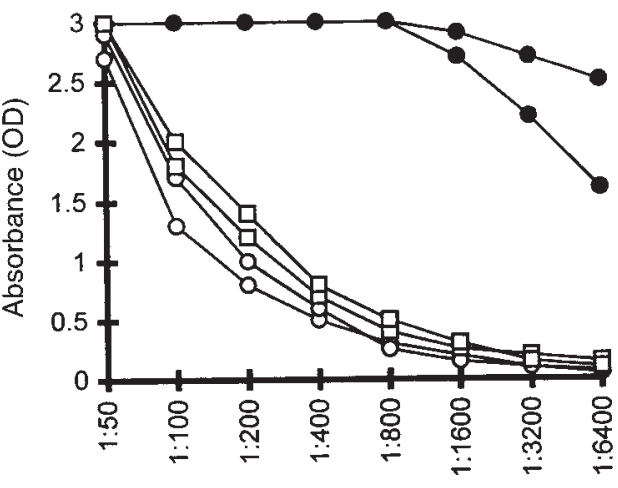

Serum dilution

\section{Figure 6}

Anti-Gal IgG response in $\alpha 1,3 G T$ KO mice is dependent on helper T cells that are activated by xenopeptides. (a) Effect of anti-CD40L antibody on anti-Gal production in mice immunized twice with pig kidney membranes: $\lg$ (filled circles) and IgM (open circles) indicate response in mice treated with anti-CD40L antibody or with control hamster IgG (filled squares, open squares), respectively. Data are from a representative mouse of 5 in each group. (b) Response in mice immunized with human/mouse hybridoma (filled circles) or with mouse/mouse hybridoma (open circles). Data represent anti-Gal activity in 3 mice of 7 with similar results in each group. (c) Response in mice that were preimmunized with $\mathrm{KLH}$, then immunized with $\mathrm{KLH}$-coupled $\mathrm{C} 3 \mathrm{H}$ mouse kidney membranes (filled circles) or with $\mathrm{C} 3 \mathrm{H}$ kidney membranes (open circles). Data are from 2 representative mice of 4 in each group. (d) Response in mice preimmunized twice with pig kidney membranes and, after 2 months, immunized twice with allogeneic mouse kidney membranes (open circles) or with pig kidney membranes (filled circles). Antibody activity before second set of immunizations is represented by open squares. Data are from 2 representative mice of 5 in each group.

this purpose we studied anti-Gal $\operatorname{IgG}$ response in mice immunized with mouse myeloma SP2/0 cells that were fused with human lymphocytes or with $\alpha 1,3 \mathrm{GT}$ KO mouse lymphocytes. Whereas SP2/0 cells express $\alpha$-gal epitopes, both $\alpha 1,3 \mathrm{GT} \mathrm{KO}$ mouse lymphocytes and human lymphocytes lack this epitope $(21,23)$. However, the human lymphocytes provide multiple genes for the synthesis of a very large variety of proteins that serve as xenoantigens in the resulting polyclonal hybridomas. Mice immunized with human/mouse hybridoma cells displayed extensive production of anti-Gal IgG, whereas mice immunized with mouse/mouse hybridoma cells had only marginal activity of this antibody (Figure 6b). Thus, expression of xenoproteins on mouse cells alowed them to induce anti-Gal IgG production when administered into $\alpha 1,3 \mathrm{GT} \mathrm{KO}$ mice.

The effect of xenopeptides on helper $T$ cells that are associated with anti-Gal IgG response was also demonstrated with KLH. $\alpha 1,3 \mathrm{GT}$ KO mice were immunized twice with KLH in order to expand helper T-cell populations that recognize KLH peptides. Subsequently, the mice were immunized twice with $\mathrm{C} 3 \mathrm{H}$ kidney membranes that expressed coupled KLH. These mice produced anti-Gal IgG, whereas immunization of the mice with $\mathrm{C} 3 \mathrm{H}$ kidney membranes that lacked coupled KLH did not elicit production of this antibody (Figure 6c). It should be stressed, however, that the activity of this antibody in mice immunized with KLH-coupled allogeneic membranes was lower than that in mice immunized with xenogeneic membranes.

The inability of allopeptides or of $\alpha$-gal epitopes to activated $\mathrm{T}$ cells that are required for anti-Gal IgG response could be further demonstrated in $\alpha 1,3 \mathrm{GT}$ KO mice that were first immunized with xenomembranes, followed by allomembranes. Mice were immunized twice by pig kidney membranes, as above, and found to produce anti-Gal IgG, as described in Figure 1b. After 2 months, the mice were immunized twice with $100 \mathrm{mg}$ kidney membranes 


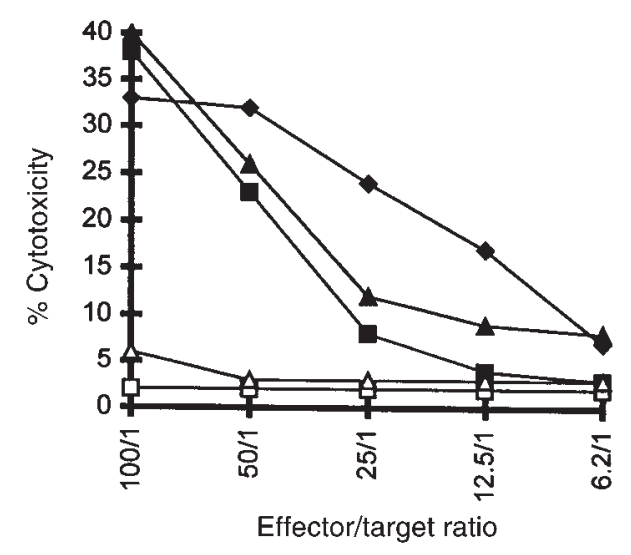

\section{Figure 7}

Destruction of cells expressing or lacking $\alpha$-gal epitopes by the ADCC mechanism with mouse splenocytes as effector cells and with mouse anti-Gal $(4 \mu \mathrm{g} / \mathrm{mL})$. Target cells studied are: BL $\sigma_{\alpha \mathrm{GT}}$ melanoma cells expressing $\alpha$-gal epitopes (filled triangles); BL6 cells lacking $\alpha$-gal epi-

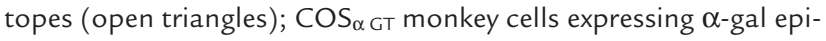
tope (filled squares); $\operatorname{COS}$ cells lacking $\alpha$-gal epitopes (open squares); pig PK15 cells (filled diamonds). Data are representative from 1 of 5 mice with similar results.

from $\mathrm{C} 3 \mathrm{H}$ mice or from pigs. As shown in Figure 6d, allogeneic kidney membranes failed to induce additional production of anti-Gal IgG in the immunized mice, whereas the xenogeneic membranes were very effective in inducing the production of this antibody. Thus, in the absence of xenoproteins in the immunizing membranes, previous exposure of the immune system to $\alpha$-gal epitopes, on xenoglycoproteins, does not confer the ability to respond to $\alpha$-gal epitopes on allogeneic kidney membranes for the production of anti-Gal IgG.

Elicited anti-Gal IgG destroys xenograft cells by ADCC. The elicited anti-Gal IgG response against $\alpha$-gal epitopes on xenografts is detrimental to the xenograft cells because this antibody mediates the destruction of any cell expressing $\alpha$-gal epitopes by the ADCC mechanism. This process could be demonstrated by using target cells expressing $\alpha$-gal epitopes and comparing their ADCC lysis to that of the same cells that, however, lack the epitope. For this purpose we compared the antiGal-mediated ADCC of BL6 and BL6 aGT mouse cells and of monkey COS and $\operatorname{COS}{ }_{\alpha \mathrm{GT}}$ cells. As shown in Figure 7, anti-Gal isolated from immunized $\alpha 1,3 \mathrm{GT}$ KO mice readily induced ADCC by splenocytes, when mouse and monkey target cells expressed $\alpha$-gal epitopes. In contrast, BL6 or COS cells that lacked $\alpha$-gal epitopes were not killed by the splenocytes, because they did not bind anti-Gal. The pig kidney cell line PK15, which expresses approximately $10^{7} \alpha$-gal epitopes/cell, also was readily killed by ADCC (Figure 7).

\section{Discussion}

The data presented in this study illustrate that $\alpha$-gal epitopes on xenografts elicit a much stronger immune response than does the same epitope on allografts. By using the experimental model of $\alpha 1,3 \mathrm{GT}$ KO mice, which mimics anti-Gal immune response in humans and monkeys $(14,18)$, we found marked expansion of anti-Gal B cells and extensive anti-Gal IgG production in response to stimulation by $\alpha$-gal epitopes on xenograft membranes. In contrast, production of this antibody in response to $\alpha$-gal epitopes on allogeneic cell membranes was marginal. Anti-Gal IgG produced in xenograft recipients is highly detrimental to cells expressing the $\alpha$-gal epitope. After binding to xenograft cells, the Fc portion of this antibody effectively interacts with $\mathrm{Fc} \gamma$ receptors on monocytes, macrophages, granulocytes, and natural killer (NK) cells. Such interaction results in destruction of xenograft cells by the ADCC mechanism, previously shown to be mediated also by human anti-Gal IgG (1). This cell lysis may be exacerbated by the binding of anti-Gal IgM molecules to the xenograft cells and complement activation. The resulting interaction of membrane-bound $\mathrm{C} 3 \mathrm{~b}$ molecules with $\mathrm{C} 3 \mathrm{~b}$ receptors on various effector cells is synergistic to anti-Gal IgG in facilitating ADCC. Moreover, formed C5a molecules recruit leukocytes to the xenograft.

Understanding these differential xenogeneic and allogeneic immune responses is based on the following observations in $\alpha 1,3$ GT KO mice: (a) whereas pig membranes can induce extensive anti-Gal IgG production, mouse membranes expressing $\alpha$-gal epitopes induce only a weak production of the IgM isotype of this antibody; (b) pig membranes, but not mouse membranes, induce expansion of B cells with anti-Gal BCR; (c) glycolipids with $\alpha$-gal epitopes induce only a weak anti-Gal IgM response and do not stimulate the proliferation and expansion of anti-Gal B cells; (d) lymphocytes in mice immunized with pig membranes proliferate in vitro in the presence of xenomembranes, but do not directly respond to $\alpha$-gal epitopes; (e) blocking of T-B cell interaction by anti-CD $40 \mathrm{~L}$ antibody prevents production of anti-Gal IgG, but not anti-Gal IgM; (f) immunization with mouse cells expressing $\alpha$-gal epitopes and xenoproteins (e.g., human/mouse hybridomas) results in anti-Gal IgG production; and (g) allogeneic kidney membranes expressing $\alpha$-gal epitopes fail to induce antiGal IgG response in mice immunized previously with xenomembranes. All these observations imply that the interaction of $\alpha$-gal epitopes on glycolipids or on allogeneic membranes with anti-Gal B cells can induce only incomplete activation of these cells, resulting in the production of IgM, but not of IgG antibodies. The immunizing membranes have to express xenoproteins in order to complete the activation process so that anti-Gal B cells are stimulated to proliferate, undergo isotype switch, and produce anti-Gal IgG.

It is well-established that induction of B-cell proliferation and of isotype switch, subsequent to the binding of nonbacterial antigens to the corresponding $\mathrm{BCR}$, are $\mathrm{T}$-dependent processes (22). The helper $\mathrm{T}$ cells required for this process must be activated to secrete cytokines, to upregulate expression of a number of adhesion/acti- 
vation molecules, and to transiently express the CD40 ligand (CD40L) that interacts with CD40 on B cells (19). This interaction is required for the production of anti-Gal IgG by B cells, as indicated by the complete inhibition of this process by anti-CD40L antibody in mice immunized with xenomembranes (Figure 6a).

It is probable that $\alpha$-gal epitopes cannot be processed and presented by class II MHC molecules on antigenpresenting cells (APC) for activation of helper T cells. This is because the endosomal compartment in APC contains $\alpha$-galactosidase that cleaves $\alpha$-gal epitopes. The absence of this enzyme in humans results in Fabry's disease (24). Moreover, carbohydrate chains are incapable of binding to class II MHC molecules (25). These considerations are in accord with our observations on the absence of in vitro proliferative response of T cells to $\alpha$-gal epitopes (Figure 5). Furthermore, initial exposure of the $\alpha 1,3 \mathrm{GT}$ KO mouse immune system to $\alpha$-gal epitopes on xenogeneic kidney membranes did not result in the expansion of a helper T-cell population that could be activated subsequently by this epitope on allogeneic kidney membranes (Figure 6d).

The data in the present study imply that the help required by anti-Gal $\mathrm{B}$ cells is provided by helper T cells that are activated by xenopeptides. The presence of numerous immunogenic peptides on xenografts is the result of 70-80 million years of separate evolution of the various mammalian orders. The continuous mutation process in all genes throughout evolution results in amino acid substitutions to the extent of $10-25 \%$ sequence differences between homologous proteins in pigs, mice, and humans (26). For this reason, many pig peptides contain species-specific sequences that are immunogenic (i.e., recognized by $\mathrm{T}$-cell receptors [TCRs] on helper T cells) in mice or in humans, upon processing and presentation by APC.

Based on these considerations, the following mechanism is suggested for explaining the stimulatory role of species-specific pig xenopeptides in the induction of anti-Gal IgG response. Pig glycoproteins that have $\alpha$-gal epitopes bind to BCR of anti-Gal B cells (Figure 8a). This interaction induces the uptake of such molecules by $\mathrm{B}$ cells that function as APC. The subsequent processing of pig glycoproteins by these $\mathrm{B}$ cells results in the presentation of many different immunogenic pig xenopeptides that interact with TCRs on some of the adjacent helper $\mathrm{T}$ cells and activate these $T$ cells. These activated helper $\mathrm{T}$ cells provide the help required by anti-Gal B cells to complete their activation process by inducing their proliferation, isotype switch, and somatic mutations (19, 22). This strong immune response ultimately results in the generation of plasma cells that produce large amounts of high-affinity anti-Gal IgG molecules.

Activation of anti-Gal B cells cannot effectively occur in $\alpha 1,3 \mathrm{GT}$ KO mice immunized with mouse allogeneic membranes expressing $\alpha$-gal epitopes. Allograft donors and recipients differ from each other mainly in $\mathrm{MHC}$ molecules and in minor transplantation antigens. Class I MHC molecules express only 1 carbohydrate chain, which may have the $\alpha$-gal epitope (27). Therefore, the likelihood for an anti-Gal B cell to present processed immunogenic allopeptides to an adjacent $T$ cell with the corresponding TCR is low. Without effective activation of helper T cells, anti-Gal B cells that engage $\alpha$-gal epitopes on alloglycoproteins do not receive sufficient $T$ cell help for completion of their activation process (Figure $8 \mathrm{~b})$. Such B cells may produce only low amounts of anti-Gal IgM molecules. The immunogenicity of allogeneic membranes could be changed by coupling of KLH. Mice that were preimmunized with KLH and subsequently immunized with KLH-coupled allogeneic kidney membranes, produced anti-Gal IgG (Figure 6c). This response was probably associated with activation of helper $\mathrm{T}$ cells by processed KLH peptides. This response was lower than that observed with pig membranes, because the repertoire of activated $T$ cells was limited to those recognizing relatively few $\mathrm{KLH}$ xenopeptides. Moreover, KLH does not express $\alpha$-gal epitopes, as do various xenoglycoproteins. Thus, most of the KLH molecules that were internalized into antiGal B cells were those that were randomly coupled to alloglycoproteins that have the $\alpha$-gal epitope.

The mechanism described above also explains the lack of immune response to blood group antigens on allografts in the accommodation state of patients transplanted with ABO-incompatible kidneys (9-11). In these patients, anti-blood group antibodies are a

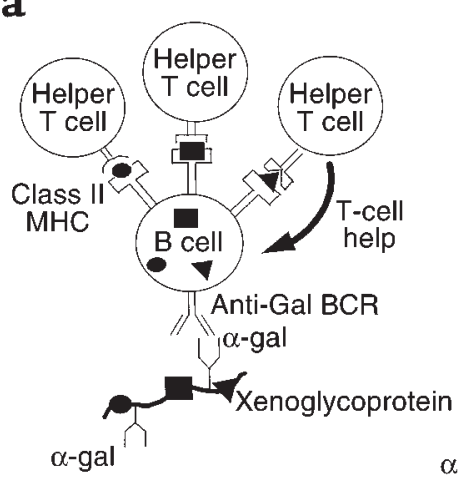

b

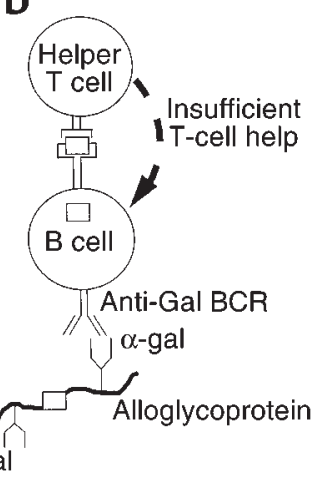

\section{Figure 8}

Proposed mechanism for the differential immune response to $\alpha$-gal epitopes on xenografts and allografts. (a) Activation of anti-Gal B cells by xenoglycoproteins expressing $\alpha$-gal epitopes and containing multiple immunogenic xenopeptides (filled circles, filled squares, filled triangles). The xenoglycoproteins are internalized by the anti-Gal B cell, subsequent to interaction of $\alpha$-gal epitopes with anti-Gal BCR. The processed xenopeptides are presented in association with class II $\mathrm{MHC}$ molecules and effectively activate many helper $\mathrm{T}$ cells with the corresponding TCR specificities. These activated T cells provide the help required by the $B$ cell to complete its activation and undergo proliferation, isotype switch, and affinity maturation for the ultimate production of high-affinity anti-Gal IgG. (b) The processing and presentation of relatively few immunogenic allopeptides (open squares) on allograft glycoproteins with $\alpha$-gal epitopes results in insufficient helper $\mathrm{T}$-cell activation. Thus, the anti-Gal $\mathrm{B}$ cell fails to complete its activation and to differentiate into a plasma cell producing IgG molecules. 
removed by plasmapheresis to prevent hyperacute rejection. In addition, these patients are splenectomized to decrease antibody production. After transplantation, the level of these antibodies returns to normal because of the ongoing $\mathrm{T}$-independent antigenic simulation by gastrointestinal bacteria $(9-11,28)$. However, as with the case of $\alpha$-gal epitopes on alloglycoproteins, the protein portion of glycoproteins expressing blood group antigens contains only relatively few amino acid sequences that differ between donor and recipient. This low immunogenicity of allopeptides, processed and presented by the recipient's APC, is insufficient for effective helper T-cell activation. Therefore, the immune system of most recipients does not respond to the carbohydrate blood group antigens on the allograft, and the activity of anti-blood group antibodies in most recipients does not increase above pretransplantation levels (9-11). In contrast, the immune response to $\alpha$-gal epitopes on xenografts is very strong and cannot be prevented without the complete paralysis of the immune system. Thus, it is probable that accommodation of pig xenografts may not be feasible under conditions that enable interaction of the recipient's anti-Gal with $\alpha$ gal epitopes on the xenograft.

\section{Acknowledgment}

We thank Aron Thall and John B. Lowe for providing us with the $\alpha 1,3 \mathrm{GT}$ KO mice. A.S. Chong's work is supported by NIH grants AI-43631 and AI-34061.

1. Galili, U. 1993. Interaction of the natural anti-Gal antibody with $\alpha$-galactosyl epitopes: a major obstacle for xenotransplantation in humans. Immunol. Today. 14:480-482.

2. Good, A.H., et al. 1992. Identification of carbohydrate structures that bind human antiporcine antibodies: implications for discordant xenografting in humans. Transplant. Proc. 24:559-562.

3. Sandrin, M.S., Vaughan, H.A., Dabkowski, P.L., and McKenzie, I.F.C. 1993. Anti-pig IgM antibodies in human serum react predominantly with Gal $(\alpha 1-3)$ Gal epitopes. Proc. Natl. Acad. Sci. USA. 90:11391-11395.

4. Cozzi, E., et al. 1997. Effect of transgenic expression of human decayaccelerating factor on the inhibition of hyper acute rejection of pig organs. In Xenotransplantation. 2nd edition. D.K.C. Cooper, E. Kemp, J.L. Platt, and D.J.G. White, editors. Springer. Heidelberg, Germany. 665-682.

5. Byrne, G.W., et al. 1997. Transgenic pigs expressing human CD59 and decay accelerating factor produce an intrinsic barrier to complement mediated damage. Transplantation. 63:149-158.

6. Xu, Y., et al. 1998. Removal of anti-porcine natural antibodies from human and nonhuman primate plasma in vitro and in vivo by Gal $\alpha 1$ 3Galß1-4Glc-X immunoaffinity column. Transplantation. 65:172-179.

7. Galili, U., Tibell, A., Samuelsson, B., Rydberg, L., and Groth, C.G. 1995. Increased anti-Gal activity in diabetic patients transplanted with fetal porcine islet cell clusters. Transplantation. 59:1549-1556.

8. Stone, K.R., et al. 1998. Porcine cartilage transplants in cynomolgus monkey. III. Transplantation of $\alpha$-galactosidase treated porcine cartilage. Transplantation. 65:1577-1583.

9. Reding, R., et al. 1987. Early post-operative monitoring of natural anti$\mathrm{A}$ and anti-B isoantibodies in $\mathrm{ABO}$ incompatible living donor renal allografts. Transplant. Proc. 19:1989-1990.

10. Alexandre, G.P., et al. 1987. Present experiences in a series of 26 ABOincompatible living donor renal allografts. Transplant. Proc. 19:4538-4542.

11. Slapak, M., Digard, N., Ahmed, M., Shell, T., and Thompson, F. 1990. Renal transplantation across the $\mathrm{ABO}$ barrier-A 9-year experience. Transplant. Proc. 22:1425-1428.

12. Bach, F.H., Turman, M.A., Vercellotti, G.M., Platt, J.L., and Dalmasso, A.P. 1991. Accommodation: a working paradigm for progressing toward clinical discordant xenografting. Transplant. Proc. 23:205-207.

13. Bach, F.H., et al. 1997. Accommodation of vascularized xenografts: expression of "protective genes" by donor endothelial cells in a host Th2 cytokine environment. Nat. Med. 3:196-204.

14. Thall, A.D., Maly, P., and Lowe, J.B. 1995. Oocyte Gal 1 1,3Gal epitopes implicated in sperm adhesion to the zone pellucida glycoprotein $\mathrm{ZP} 3$ are not required for fertilization in the mouse. J. Biol. Chem. 270:21437-21440

15. Galili, U., Macher, B.A., Buehler, J., and Shohet, S.B. 1985. Human natural anti-- galactosyl IgG. II. The specific recognition of $\alpha(1 \rightarrow 3)$-linked galactose residues. J. Exp. Med. 162:573-582.

16. Egge, H., Kordowicz, M., Peter-Katalinic, J., and Hanfland, P. 1985. Immunochemistry of $\mathrm{I} / \mathrm{i}$-active oligo- and polyglycosylceramides from rabbit erythrocyte membranes. J. Biol. Chem. 260:4927-4935.

17. Galili, U., LaTemple, D.C., and Radic, M.Z. 1998. A sensitive assay for measuring $\alpha$-gal epitope expression on cells by a monoclonal anti-Gal antibody. Transplantation. 65:1129-1132.

18. LaTemple, D.C., and Galili, U. 1998. Adult and neonatal anti-Gal response in knock-out mice for $\alpha 1,3$ galactosyltransferase. Xenotransplantation. 5:191-196.

19. Noelle, R.J., et al. 1992. A $39 \mathrm{kDa}$ protein on activated helper T cells binds CD40 and transduces signals to cognate activation of B cells. Proc. Natl. Acad. Sci. USA. 89:6550-6554.

20. Larsen, R.D., et al. 1989. Isolation of a cDNA encoding murine UDPgalactose: $\beta \mathrm{D}$-galactosyl-1,4-N-acetyl-D-glucosaminide $\alpha$-3-galactosyltransferase: expression cloning by gene transfer. Proc. Natl. Acad. Sci. USA. 86:8227-8231.

21. Gorelik, E., Duty, L., Anaraki, F., and Galili, U. 1995. Alterations of cell surface carbohydrates and inhibition of metastatic property of murine melanomas by $\alpha 1$,3galactosyltransferase gene transfection. Cancer Res. 55:4168-4173.

22. Snapper, C.M., and Finkelman, F.D. 1999. Immunoglobulin class switching. In Fundamental immunology. 4th edition. W.E. Paul, editor. Lippincott-Raven Publishers. Philadelphia, PA. 831-861.

23. Galili, U., Shohet, S.B., Kobrin, E., Stults, C.L.M., and Macher, B.A. 1988. Man, apes, and Old World monkeys differ from other mammals in the expression of $\alpha$-galactosyl epitopes on nucleated cells. J. Biol. Chem. 263:17755-17762.

24. Desnick, R.J., Ioannou, Y.A., and Eng, C.M. 1995. $\alpha$-galactosidase A deficiency: Fabry disease. In The metabolic and molecular basis of inherited diseases. 7 th edition. C.R. Scriver, A.L. Beaudet, W.S. Sly, and D. Valle, editors. McGraw-Hill. New York, NY. 2741-2784.

25. Ishioka, G.Y., et al. 1993. Major histocompatibility complex class II association and induction of $\mathrm{T}$ cell responses by carbohydrates and glycolipids. Springer Semin. Immunopathol. 15:293-309.

26. Wilson, A.C. 1985. The molecular basis of evolution. Sci. Am. 253:164-173.

27. Lopez de Castro, J.A., Strominger, J.L, Strong, D.M., and Orr, H.T. 1982. Structure of cross reactive human histocompatibility antigens HLA-A28 and HLA-A2: possible implications for generation of HLA polymorphism. Proc. Natl. Acad. Sci. USA. 79:3813-3817.

28. Springer, G.F., and Horton, R.E. 1969. Blood group isoantibody stimulation in man by feeding blood group-active bacteria. J. Clin. Invest. 48:1280-1291. 